\title{
Superhydrophilic and solvent resistant coatings on polypropylene fabrics by a simple deposition process
}

Jason Fang, Antonios Kelarakis, Luis Estevez, Yue Wang, Robert Rodriguez, Emmanuel P Giannelis*

Department of Materials Science and Engineering, Cornell University, Bard Hall, Ithaca, New York, 14853

* To whom correspondence should be addressed. Email: epg2@cornell.edu

Keywords: multilayer deposition, plasma treatment, superhydrophilicity, modified nanosilica

\begin{abstract}
A simple yet general coating method to plasma treated polymeric substrates is presented. The method is based on electrostatic interactions between the surface functionalized nanoparticles and the charged substrate and leads to stable and solvent resistant multilayer coatings. The coatings render polypropylene (PP) hydrophilic and in the case of PP fabric superhydrophilic. The superhydrophilicity is attributed to the topography and increased roughness of the fabric compared to a planar, smooth substrate.
\end{abstract}


Coating technologies are continuously being developed in an attempt to meet a diverse range of very specific requirements and applications ${ }^{1}$. Full or partial coatings are applied to surfaces for a number of different reasons including aesthetic or functional finishes and protective layers. Current trends in this field have focused on introducing nanoparticles to coating formulations ${ }^{2,3}$. In this report, we present a simple deposition process using functionalized $\mathrm{SiO}_{2}$ nanoparticles on plasma treated polypropylene (PP) fabrics. As a control experiment, deposition on planar PP substrates is also demonstrated. The electrostatic attraction between the functionalized nanoparticles and the charged plastic substrates imparts stability and durability to the coatings. Judicious selection of the functional groups grafted to the nanoparticles and optimization of their charge density leads to coatings exhibiting hydrophilicity (superhydrophilicity in the case of the PP fabric). To the best of our knowledge this is the first demonstration of a superhydrophilic PP fabric. While, we focus here on a very specific system, the approach is general and applicable to a wide range of substrate-particle combinations.

Plasma treated polypropylene, PP, was chosen as a model substrate, since plasma treatment leads conveniently to the formation of various surface groups ${ }^{4,5}$ without sacrificing any of its bulk properties. Silica nanoparticles were used because their surface chemistry and charge density can be fine-tuned, enabling exquisite control of the electrostatic interactions between the coating and the substrate ${ }^{6,7}$. The silica nanoparticles used here were treated with N-Trimethoxysilylpropyl$\mathrm{N}, \mathrm{N}, \mathrm{N}$-trimethylammonium chloride. The presence of ammonium groups on the surface renders the functionalized nanoparticles positively charged. They remain well dispersed in water within a wide $\mathrm{pH}$ range without any tendency for agglomeration as measured by dynamic light scattering. The $\zeta$ potential of the nanoparticles was 36.4 and $21.5 \mathrm{mV}$ for $\mathrm{pH} 4$ and 7 , respectively. 
$\mathrm{SiO}_{2}$ nanoparticles can be readily deposited onto plasma treated PP (fabric or planar substrate). In contrast, $\mathrm{SiO}_{2}$ nanoparticles regardless of their surface functionalization or charge adhere weakly to untreated PP (S. I. Figure 1). Systematic variation of the plasma treatment protocol revealed that it plays only a minor role in the process which is governed instead by the charge density of the nanoparticles.

Deposition of nanoparticles with $\zeta=36.4 \mathrm{mV}$ to either fabric or planar substrate (Figure $1 \mathrm{a}$ and $\mathrm{b}$, respectively) results in a monolayer coating with a patchy coverage. On the other hand, a multilayer coating on both PP substrates is obtained when nanoparticles with $\zeta=21.5 \mathrm{mV}$ are used. The TEM picture shown in Figure 2a suggests a complete and uniform coverage along the periphery of the fiber after a single coating cycle (immersion to the aqueous nanoparticle suspension, solvent evaporation followed by repeated rinsings in water). Moreover, crosssectional TEM imaging indicates the formation of a multilayer coating with a mean value of four particle layers in thickness after a single coating, as shown in Figures $2 b$ and $2 c$ for fabric and planar substrates, respectively. Given that the functionalized nanoparticles do not show any tendency for agglomeration in solution, we believe that the multilayer coating consists of discrete nanoparticles and is not due to packing of larger aggregates. The moderate magnitude of the zeta potential $(\zeta=21.5 \mathrm{mV})$ of the nanoparticles is critical for the simultaneous formation of a multi layer (rather than a monolayer) and the good coating quality. Uncharged nanoparticles tend to aggregate; low charge density nanoparticles result in weak adhesion, while high $\zeta$ values induce strong particle-particle repulsive forces, ultimately inhibiting efficient coating. In an attempt to evaluate their stability and durability, the multi layer coatings were subjected to ultrasonic field 
while being suspended in four different solvents (water, acetone, ethanol and THF). As shown in S.I. Figure 2 even after 1 hour of sonication while immersed in the solvents, the coatings resist detachment and are virtually unchanged.

The series of images shown in Figure 3i depict the profiles of an impinging water droplet onto various plasma treated PP surfaces. In the case of the planar, smooth substrate deposition of $\mathrm{SiO}_{2}$ nanoparticles leads to a hydrophilic surface with a water advancing contact angle of $28^{\circ}$ compared to $71^{\circ}$ for the bare surface. The water contact angle is $62^{\circ}$ for the coating obtained using higher $\zeta$ potential nanoparticles. There already exists extensive literature on altering the wetting characteristics of planar substrates. Various approaches include deposition of thin films of titania $^{8}$, hollow silica spheres ${ }^{9}$, silica nanoparticles ${ }^{10,11}$ or titania-silica alternating multilayers ${ }^{12}$ based on layer-by-layer assembly. It has been already shown that a minimum film thickness is required for obtaining superhydrophilic coatings necessitating multiple deposition cycles $^{13}$.

In contrast to the planar substrate, the coated fabric surface exhibits superhydrophilic characteristics showing virtually $0^{\circ}$ advancing water contact angle in less than 0.07 s (S.I. Figure 3). The corresponding contact angles for the monolayer coated and the bare fabric are $45^{\circ}$ and $120^{\circ}$, respectively (Figure 3). Wetting of a textured surface from a given solvent critically depends upon the surface-solvent chemical affinity as well as certain topological characteristics of the surface such as roughness and porosity ${ }^{14,15}$. The observed difference in wetting behavior between the planar substrate and the fabric is a direct consequence of the topography of the latter that gives rise to fundamental differences in the roughness between the two substrates ${ }^{16}$. We 
note that in the case of PP fabrics, approaches such as plasma treatment ${ }^{17}$ or grafting of vinylpyridine chains ${ }^{18}$ do not confer superhydrophicity. We believe our approach is the first demonstration of a superhydrophilic PP fabric.

In order to further explore the coating mechanism of our system we followed an identical deposition protocol using instead an acidified colloidal dispersion $(\mathrm{pH}=4)$ of the unmodified silica nanoparticles. After a single coating cycle the unmodified nanoparticles form an incomplete, patchy monolayer (S.I. Figure 4), which does not improve hydrophilicity. This result underlines the key role of the functional groups on the nanoparticles to the coating quality in determining the charge density, the layer thickness and topography and ultimately altering the intrinsic wetting characteristics of the coating. Note that the water contact angle of unmodified planar silica surface is $20^{\circ}{ }^{19}$, e.g. substantially higher than the values for superhydrophilic silica based coating described here.

Lastly, we note that the cationically modified silica nanoparticles can effectively support sequential deposition of opposite charged nanoparticles. To demonstrate this opportunity, negatively charged carbon black particles were deposited on silica coated fabric as clearly shown in Figure 4. Current work is focusing on multilayer (ABAB...) deposition of opposite charged nanoparticles to create highly tuned, functional surfaces.

In summary, we report on a simple coating approach that allows the formation of a multilayer of nanoparticles on charged substrates after a single-step deposition. The method relies on electrostatic substrate-particle and particle-particle interactions that largely depend upon the 
charge density of nanoparticles. The resulting multilayer coatings show remarkable stability in water and other solvents. In addition the coatings render the substrate hydrophilic (superhydrophilic in the case of the PP fabric due to the topography and increased roughness of that substrate).

\section{Acknowledgements}

This publication was based on work supported by Award No KUS-C1-018-02, made by King Abdullah University of Science and Technology (KAUST).

\section{Experimental Section}

Cationically modified Silica nanoparticles. Colloidal silica Ludox HS-30 with a mean diameter of $18 \mathrm{~nm}$ was purchased from Sigma Aldrich. 3 gram of colloidal silica was diluted with deionized water $(30 \mathrm{~mL})$ and sonicated for $30 \mathrm{~min}$. A concentrated solution of $\mathrm{HCl}(1 \mathrm{~N})$ was added to the dispersion followed by the addition of $3.2 \mathrm{~g}$ of N-Trimethoxysilylpropyl-N,N,Ntrimethylammonium chloride (50 wt \%, Gelest). The mixture was stirred at $60{ }^{\circ} \mathrm{C}$ for $10 \mathrm{~min}$. $\mathrm{NaOH}(0.1 \mathrm{M})$ was added to adjust the $\mathrm{pH}$ to $\sim 5$ and the mixture was stirred continuously at 60 ${ }^{\circ} \mathrm{C}$ for $24 \mathrm{~h}$ to complete the reaction. Subsequently, the suspension was dialyzed in deionized water using SnakeSkin tubing (3.5k MWCO, Pierce) for $48 \mathrm{~h}$.

Plasma treated Polypropylene : Industrial spun-bounded polypropylene nonwoven fabric (0.9 $\mathrm{g} / \mathrm{cm}^{3}, 240 \pm 20 \mu \mathrm{m}$ thick) with a melt flow index of $36.0 \mathrm{~g} / 600 \mathrm{~s}$ supplied by Kimberly-Clark Company, was used as substrate. The substrate was treated with $\mathrm{Ar} / \mathrm{O}_{2}(50 / 50)$ mixed gas plasma under $234 \mathrm{~W}$ for $2 \mathrm{~min}$. Based on X-ray photoelectron spectroscopy the oxygen/carbon ratio of 
the surface after plasma treatment was 0.12 . The planar PP substrate was provided by Bamberger Polymers (product Bapolene 4082) and was plasma treated under $75 \mathrm{~W}$ for 1 min using a Glen 1000 Resist Strip apparatus, (oxygen/carbon ratio of the plasma treated surface was 0.12 ).

Surface Contact Angle: Static and dynamic advancing contact angle measurements were carried out by means of a VCA Optima XE apparatus. The water droplets (deionized water from Millipore purification system, specific conductance $0.05 \mu \mathrm{S} / \mathrm{cm}, \mathrm{pH} 5.5$, droplet volume $0.5 \mu \mathrm{L}$ ) were monitored by a CCD camera and analyzed by standard drop-shape analysis methods.

Zeta potential: Electrophoretic measurements were made using a Malvern Zetasizer Nano-ZS (Malvern Instruments, England) package which includes a $4 \mathrm{~mW}$ He-Ne laser operating at $\lambda=$ $633 \mathrm{~nm}$. Dust-free solutions were obtained more than $12 \mathrm{~h}$ before measurement by filtration through Nylon membrane filters with a pore size of $(0.2 \sim 0.45 \mu \mathrm{m})\left(\mathrm{GE}\right.$ Nylon ${ }^{\circledR}$ Syringe Filter).

Scanning Electronic Microscopy (SEM): SEM measurements were performed on a Keck Field Emission Scanning Electron Microscope (FE-SEM), LEO 1550 model.

Transmission electron microscopy (TEM): TEM imaging was performed on FEI Tecnai T12 using microtomed epoxy-embedded ultrathin samples. 


\section{Figures}

Figure 1. TEM images of the plasma treated PP surfaces coated with a monolayer of cationically modified silica nanoparticles $(\zeta=36.4 \mathrm{mV})$ after deposition on a fabric (a) and planar (b) substrate.

Figure 2. TEM images of the plasma treated PP surfaces coated with a multilayer of cationically modified silica nanoparticles $(\zeta=21.5 \mathrm{mV})$ after a one-step deposition on a fabric $(\mathrm{a}$ and $\mathrm{b})$ and planar (b) substrate.

Figure 3. i) Profiles of advancing water contact angle of various plasma treated polypropylene substrates, both fabric and planar: a) uncoated substrates; b and c) coated with silica nanoparticles with $\zeta=36.4 \mathrm{mV}$ and $21.5 \mathrm{mV}$, respectively. ii) Structure- properties relationships in plasma treated polypropylene fabrics.

Figure 4. SEM image of the plasma treated polypropylene fabric after the first layer deposition of cationically modified $\mathrm{SiO}_{2}$ nanoparticles (left side of Figure 4), followed by sequential deposition of negatively charged carbon black nanoparticles (right side of Figure 4). 


\section{References}

1. “Coating Technology Handbook” edited by D. Satas, A. A. Tracton, Marcel Dekker Inc., New York, 2001.

2. "Nanotechnology Applications in Coatings" edited by R. H. Fernando, L.-P. Sung, ACS Symposium Series, vol. 1008, 2009.

3. "Smart Coatings II" edited by T. Provder, J. Baghdachi, ACS Symposium Series, vol. $1002,2009$.

4. E. M. Liston, L. Martinu, M. R. Wertheimer, J. Adhension Sci. Technol. 1993,7,1091-1127.

5. M. Strobel, M. J. Walzak, J. M. Hill, A. Lin, E. Karbashewski, C. S. Lyons, J. Adhension Sci. Technol. 1995,9,365-383.

6. E. F. Vansant, P. van der Voort, K. C. Vrancken, "Characterization and Chemical Modification of the Silica Surface" Elsevier, Amsterdam, 1995.

7. "Colloidal Silica: Fundamentals and applications" edited by H. E. Bergna, W.O. Roberts, Taylor and Francis Group, U.S.A., 2006.

8. R. Wang, K. R. Wang, K. Hashimoto, A. Fujishima, M. Chikuni, E. Kojima, A. Kitamura, M. Shimohigoshi, T. Watanabe, Nature 1997, 388, 431-432.

9. X. Liu, X. Du, J. He, Chem. Phys. Chem. 2008,9,305-309.

10. F. Wang, S. Peters, J. Cuzda, R. H. Blunk, A. P. Angelopoulos, Langmuir 2009,25,43844392.

11. X. Liu, J. He, J. Phys. Chem. C 2009 113,148-152. 
12. X. T. Zhang, O. Sato, M. Taguchi, Y. Einaga, T. Murakami, A. Fujishima, Chem. Mater. 2005,17, 696-700.

13. F. C. Cebeci, Z. Wu, L. Zhai, R. E. Cohen, M.F. Rubner, Langmuir 2006, 22, 2856-2862.

14. J. Bico, U. Thiele, D. Quere, Colloids and Surfaces A: Physicochem. Eng. Aspects 2002, 206, 41-46.

15. G. McHale, N. J. Shirtcliffe, S. Aqil, C. C. Perry, M. I. Newton, Phys. Rev. Lett. 2004,93,036102-4.

16. G. McHale, M.I. Newton, N. J. Shirtcliffe, J. Phys.:Condens. Matter. 2009, 21,464122.

17. Y. J. Hwang, M. G. Mccord, J. S. An, B. K. Kang, S. W. Park, Texile Res. J. 2005, 75,771778.

18. C. Wang, F. Yang, L.-F. Liu, Z. Fu, Y. Xue, J. Membr. Sci. 2009,345,223-232.

19. D. Lee, M. F. Rubner, R. E. Cohen, Nano Lett. 2006, 6, 2305-2312. 

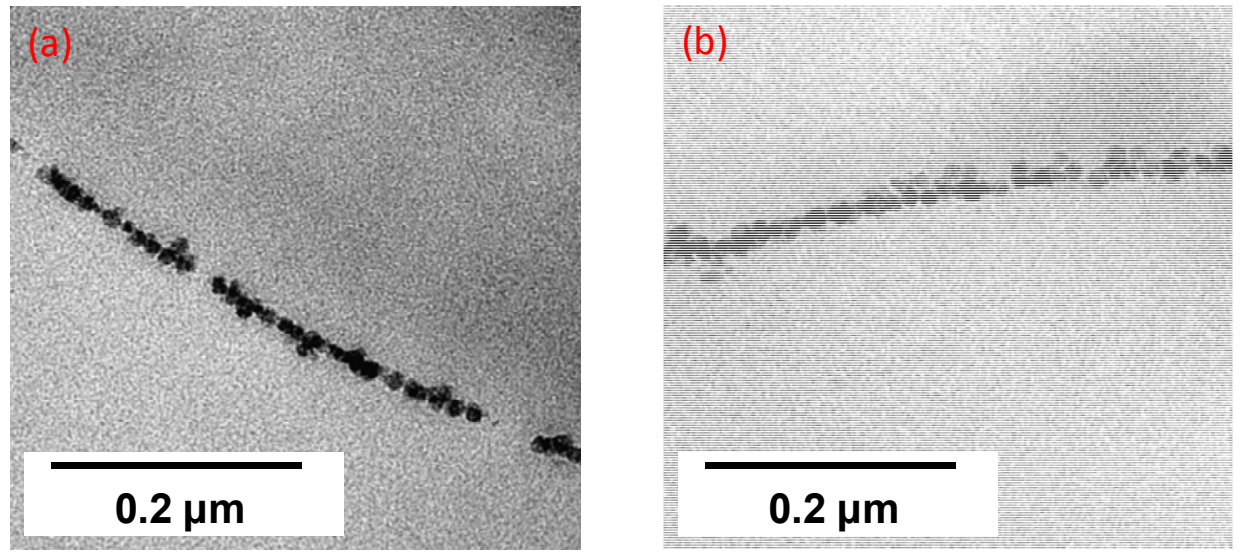

Figure 1 

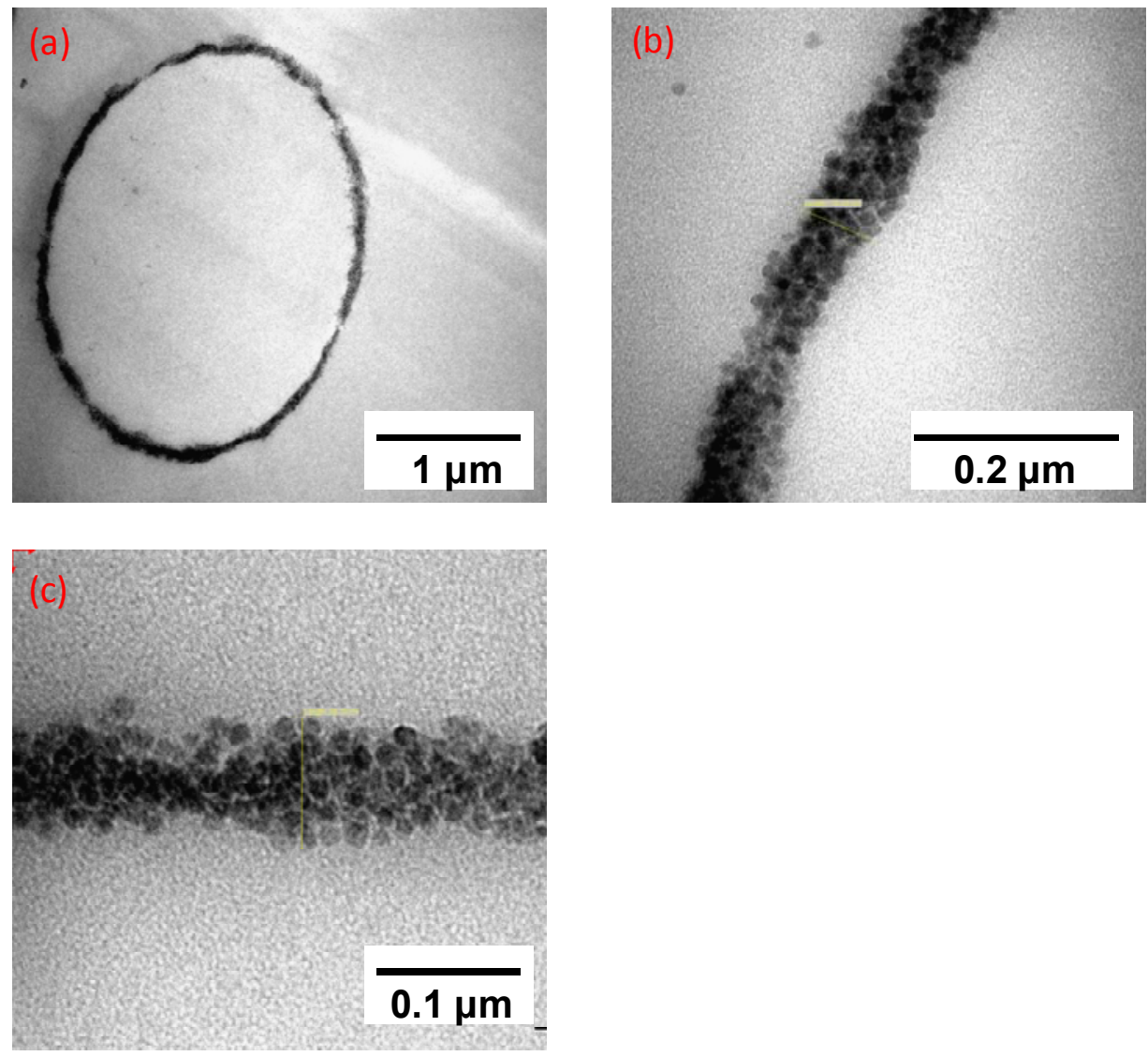

Figure 2 

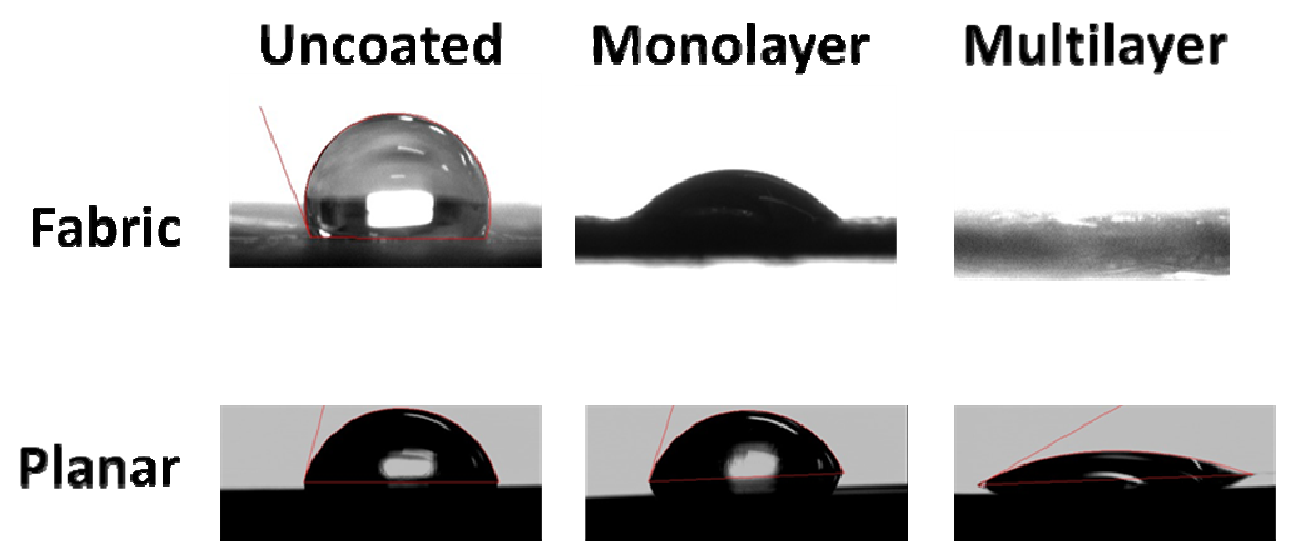

ii

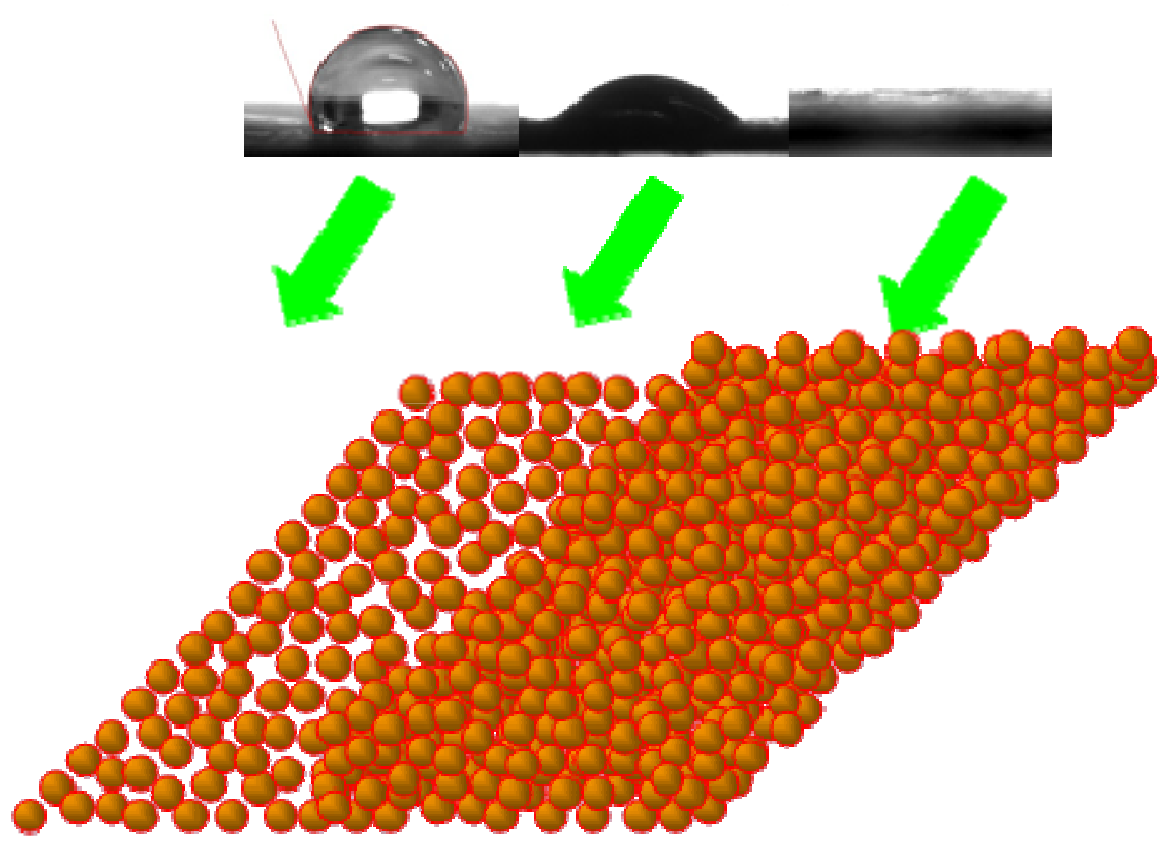

Figure 3 


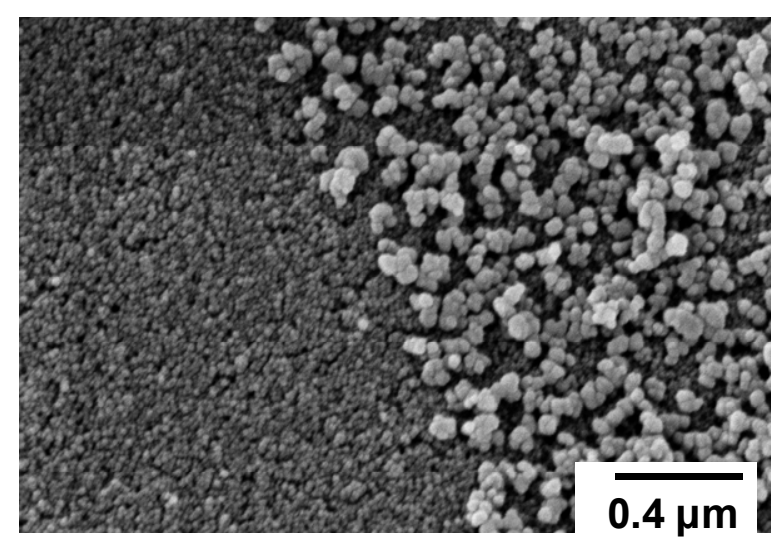

Figure 4 